\title{
Babesia parasitemia causing splenic infarction: A review of the literature
}

\author{
Darren Wong* \\ Division of Infectious Diseases, Albert Einstein College of Medicine, Bronx, NY, USA
}

Received: May 14, 2016

DOI: $10.5430 /$ crim.v3n3p78
Accepted: July 6, 2016

URL: http://dx.doi.org/10.5430/crim.v3n3p78

\begin{abstract}
Babesia remains an underappreciated and under-diagnosed tick-borne pathogen which can lead to significant morbidity and mortality. We present an uncommon manifestation of parasitemia causing splenic infarction. The pathophysiology is reviewed highlighting the significant challenges in diagnosis and treatment of this unique pathogen.
\end{abstract}

Key Words: Babesia, Spleen, Splenic infarction

\section{INTRODUCTION}

Babesia is an under-recognized hemoparasite encompassing multiple strains which afflict a wide range of mammalian and avian species. In humans, Babesia can occur as a co-infection with other tick-borne pathogens such as Lyme, Ehrlichia, and Anaplasma. Notably, Babesia has a diverse range of clinical manifestations, and remains a particular challenge in both diagnosis, as well as treatment. Splenic infarction, and even splenic rupture, is an uncommon complication of Babesia parasitemia and requires a high index of suspicion to ensure proper diagnosis and timely initiation of therapy.

\section{Case presentation}

A 56-year-old male with a past medical history of insulindependent diabetes was admitted with 4 days of severe neck and right sided shoulder discomfort which was unrelieved by over-the-counter analgesics. He reported myalgias, fatigue, at least two days of fevers, and a history of night sweats for an unclear duration. He denied gastrointestinal or pulmonary symptoms and had no sick contacts or weight changes. The patient was originally born in Jamaica and immigrated to the United States ten years earlier. The patient's occupational history was significant as he served as a landscaper throughout the northeastern United States, in particular in Nantucket, an island off the coast of Massachusetts, during the summer. He had no history of illicit drug use, tobacco use, or incarceration.

In the emergency room the patient's temperature was recorded at $39.4^{\circ} \mathrm{C}$. His heart rate was 88 beats per minute, blood pressure 159/88 millimeters of mercury, and respiratory rate was 20 breaths per minute. On physical exam, the patient had no appreciable cardiac murmur. He had no thrush, rashes, or skin lesions, and both pulmonary and abdominal exam were unremarkable. The patient did not have any joint effusions and extensive musculoskeletal exam did not show crepitus, reduced range of motion, or neck stiffness.

The patient's laboratory studies were significant for a white blood count $5.2 \mathrm{k} /$ cumm with $52 \%$ polymorphonuclear leukocytes and $1.5 \%$ eosinophils. Additional laboratory studies included a hemoglobin $10.7 \mathrm{~g} / \mathrm{dl}$, platelets $163 \mathrm{k} / \mathrm{cumm}$, and

${ }^{*}$ Correspondence: Darren Wong; Email: darrenww@usc.edu; Address: Division of Infectious Diseases, Albert Einstein College of Medicine, Bronx, NY, USA. 
creatinine $1.2 \mathrm{mg} / \mathrm{dl}$. Liver function studies were elevated with an AST 156 IU/L, ALT 325 IU/L, alkaline phosphatase $266 \mathrm{IU} / \mathrm{L}$, bilirubin $0.7 \mathrm{mg} / \mathrm{dl}$, albumin $2.8 \mathrm{~g} / \mathrm{dl}$, and lactose dehydrogenase elevation of 767 U/L. Additionally, HIV antibody was negative and both urinalysis and chest radiography were unremarkable. Blood and urine cultures were without growth. Due to the patient's nonspecific systemic symptoms and transaminitis, a CT scan of the abdomen was performed in the emergency room. The CT scan was significant for multiple hypodense lesions in the spleen which were suggestive of an infectious focus or infarction (see Figure 1).

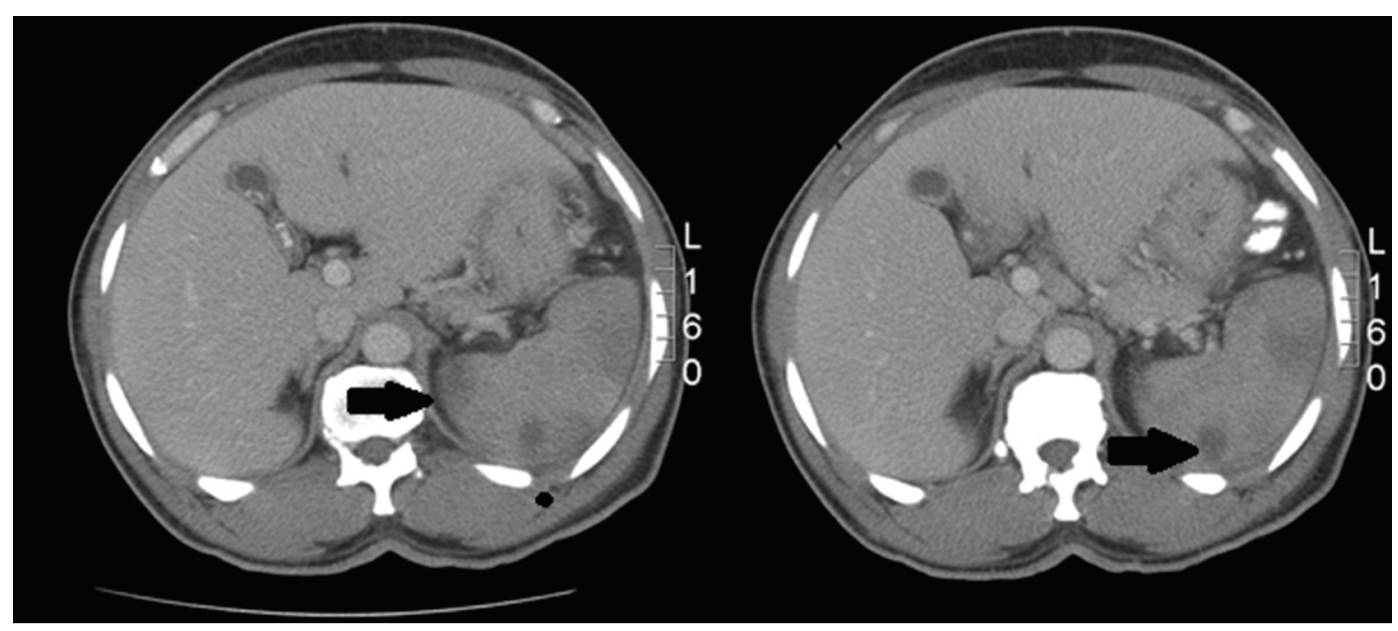

Figure 1. Cholelithiasis with one or more stones in the region of the gallbladder neck or cystic duct. No definite bile duct dilatation. Multiple hypodense lesions in the spleen. These could represent foci of infection, splenic infarcts, or masses. Probable mild hepatomegaly. Diffuse mild thickening of the colonic wall suggesting a nonspecific pan-colitis

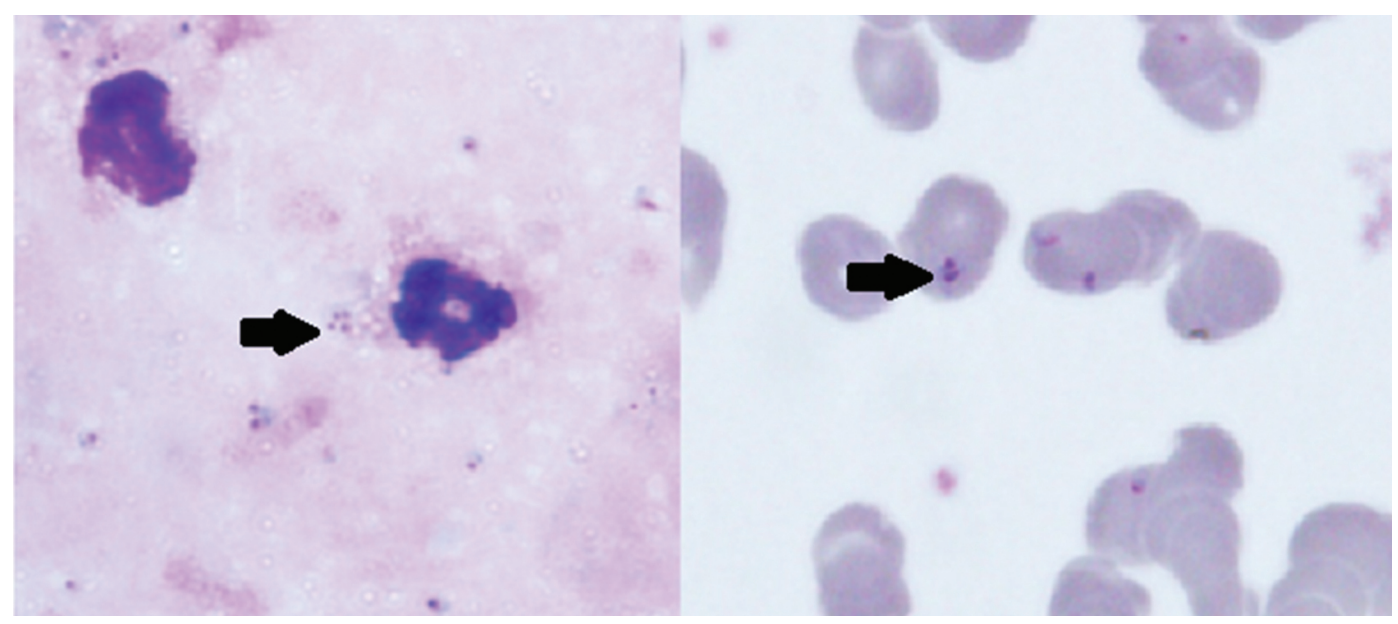

Figure 2. Thick (left) and thin (right) peripheral blood smear identifying Babesia hemoparasites at $1,000 \times$ magnification

The patient received empiric antibacterial therapy with ciprofloxacin and metronidazole in the ER which were discontinued pending diagnostic workup. A transthoracic echocardiogram did not show evidence of valvular disease. Blood smears were sent which returned positive for Babesia species at $1.5 \%$ parasitemia (see Figure 2). The patient was started on treatment with azithromycin and atovaquone for ten days. He defervesced within 48 hours of treatment. However, his lactose dehydrogenase and transaminases were slow to resolve and doxycycline was added to the patient's an- timicrobial regimen on hospital day 5 for empiric therapy of potential tick-borne co-infections pending confirmatory studies. The patient subsequently developed a left facial palsy and Lyme serology with western blot returned positive. The patient completed a 10-day course of Babesia therapy and blood smears cleared of parasitemia. He also completed a 28-day course of doxycycline for Lyme disease and at follow up, his facial palsy had near complete resolution and his systemic symptoms had fully resolved. 


\section{DisCussion}

Babesia is a tick-transmitted hemoparasite which was first discovered during study of febrile hemoglobinuria in Romanian cattle in 1888. There have been more than 100 strains identified which have been found to cause infections in a wide variety of mammalian and avian species. The first reported human case of Babesia infection occurred in 1956 in a splenectomized patient which highlights the critical role the spleen plays in pathogenesis. Babesia has since been recognized as a potentially fatal zoonotic infection with 7 strains found to be capable of causing human disease. The most common species domestically is Babesia microti, a strain endemic to the eastern United States.

The parasitic lifecycle of Babesia relies on Ixodid ticks as a vector. Ticks ingest Babesia gametocytes while feeding from an infected host. After ingestion parasitic replication occurs in the tick gut before migration into salivary glands. At a successive blood-meal larval sporozoites are injected into a human host and subsequently invade red blood cells where they replicate asexually via budding into either merozoites capable of persistent cycles of red-blood cell invasion or gametocytes which are the tick-infective form. ${ }^{[1]}$ Although transmission via direct inoculation from a tick is by far the most common route of transmission, contaminated blood transfusion, and rarely transplacental transmission have been documented. Due to tick-dependent transmission patterns, peak acquisition of disease occurs from May to September with incubation periods lasting from five to thirty-three days. However, longer incubation periods are common in transfusion derived infection and in immunocompromised hosts. ${ }^{[1]}$

Babesia infections can present with a range of symptoms. Immunocompetent individuals may be asymptomatic and typically have low level parasitemia. They classically present with nonspecific flu-like symptoms, headaches, chills, sweats, and myalgias which may resolve after several weeks. Immunocompromised patients are often much more severely infected and can reach levels of parasitemia of up to $80 \%$. Fevers greater than $40^{\circ} \mathrm{C}$, severe anemia with hemolysis, extreme weakness, and even central nervous system involvement, respiratory failure, or congestive heart failure have been described. Independent risk factors for severe disease include splenomegaly, advanced age, and immunosuppression, particularly HIV ${ }^{[1]}$ Mortality for B. micoti is about $5 \%$; however, B. divergens infection may be significantly higher reaching up to $42 \%$ in asplenic patients due to severe hemolysis. ${ }^{[1]}$

Diagnosis of Babesia infection has principally been via visualization in Giemsa stained blood smears. Serial smears may be required in early disease, especially in immunocom- petent individuals who typically have a low parasite burden. Therefore, knowledge of disease prevalence within regional tick populations may be valuable. For example, our patient's exposure was likely while he was working outdoors in the Nantucket region where Babesia is present in approximately 50\% of ticks. Elevated transaminases, hyperbilirubinemia, a normochromic normocytic anemia, thrombocytopenia, and an elevated lactic dehydrogenase are all nonspecific signs that may be suggest Babesia infection. B. microti can also be diagnosed serologically with an immunoflurourescent antibody test. Antibody tests possesses a sensitivity of $88 \%-96 \%$. However, antibody titers may persist for 13 months to up to 6 years thereby limiting their utility. ${ }^{[2]}$ Similarly, there is a delay in seroconversion following infection, especially in immunocompromised hosts. Polymerase-chain reaction testing is also available and highly specific; however, it cannot differentiate between viable and non-viable organisms which limits the utility in assessing cure.

Splenic infarction is a rare, but reported complication of Babesia infection. Similar to malaria, Babesia can cause infarctions even with low level parasitemia. To date only two cases of splenic infarction due to Babesia have been reported. ${ }^{[3]}$ In one case, Babesia organisms were demonstrated in autopsy findings within the spleen. However, thrombosis or embolism in the splenic vessels were not seen. ${ }^{[3]}$ In animal models, multifocal coagulative necrosis was observed in the spleen of Syrian hamsters infected with the WA1 babesial variant. The proposed mechanism is that red-blood cell lysis induces formation of microthrombi and local release of vasoactive factors leading to infarctive necrosis of splenic tissue. ${ }^{[4]}$ The prognosis of Babesia cases with splenic manifestations remains guarded. Although few cases have been described, untreated chronic Babesia infections can progress to splenic rupture requiring either splenic artery embolization or splenectomy. ${ }^{[5]}$ Additionally, spontaneous splenic rupture during the acute parasitemia phase, although rare, has also been described. ${ }^{[6]}$

Treatment of Babesia infections remain a challenge. Current treatment regimens include the combination of azithromycin and atovaquone or clindamycin and quinine for 7-10 days. Plasma exchange remains a potential option in life threatening cases with high parasitemia. There is considerable debate on whether an azithromycin/atovaquone regimen is equivalent to clindamycin/quinine which is still regarded as the standard for severe disease in Infectious Disease Society of America guidelines. ${ }^{[7]}$ However, current literature suggests that an azithromycin/atovaquone regimen may be better tolerated and more effective against Babesia species. ${ }^{[2]}$ A non-blinded comparison of both regimens in 58 patients with non-life threatening disease had a lower rate of adverse 
effects in the azithromycin/atovaquone group (15\%) in comparison to the quinine/clindamycin group (72\%). Efficacy was equivalent between groups at 3 months. ${ }^{[8]}$ Similarly, in vitro data in hamsters suggested higher suppression of parasitemia occurred with azithromycin when compared to quinine, although combinational regimens had higher efficacy than any monotherapy option. ${ }^{[9]}$ It is important to note that the treatment of immunocompromised patients including those with asplenia, severe cytopenias, and in particularly HIV remains a challenge due to the potential for relapse and a delayed clearance of parasites. In a prospective study with immunocompromised patients, DNA evidence of parasites persisted beyond 7 days in $40 \%$ and $50 \%$ of those treated with azithromycin/atovaquone and clindamycin/quinine respectively. ${ }^{[3]}$ Therefore, a longer treatment duration may be necessary in this subset of patients. PCR based diagnostic tests may be a useful adjunct to determine clearance.

Babesia is an important tick-borne pathogen whose diagnosis remains a challenge. It should not be overlooked as a potential co-infector in tick-borne infections. In addition, the potential for transfusion derived infection has been readily described and may be under-diagnosed as screening for Babesia is not standard.
Our patient presented with the rare complication of splenic infarction secondary to Babesia. He did not have evidence of endocarditis, vascular disease, or any other condition that would otherwise offer an alternative etiology for the splenic infarctions seen on abdominal imaging. The patient's rapid improvement with parasite directed treatment suggests that his symptoms were due to untreated Babesia infection.

Although patients may have self-limited disease, significant morbidity and complications can occur, especially in immunocompromised patients, and serial blood smears may be required to establish a diagnosis. Therefore, physicians should maintain a high index of suspicion for Babesia based on knowledge of regional tick populations and a careful review of patient travel histories, particularly in patients with HIV or splenectomy who are at high risk for severe disease.

\section{ACKNOWLEDGEMENTS}

The author would like to acknowledge Dr. Christina Coyle who assisted in the management and diagnostic evaluation of this patient.

\section{CONFLicts OF INTEREST Disclosure}

The authors have declared no conflicts of interest.

\section{REFERENCES}

[1] Hunfeld KP, Hildebrandt A, Gray JS. Babesiosis: recent insights into an ancient disease. Int J Parasitol. 2008; 38(11): 121937. PMid:18440005 http://dx.doi.org/10.1016/j.ijpara. 2008.03.001

[2] Weiss LM. Babesiosis in humans: a treatment review. Expert Opin Pharmacother. 2002; 3(8): 1109-15. PMid:12150690 http://dx.d oi.org/10.1517/14656566.3.8.1109

[3] Florescu D, Sordillo PP, Glyptis A, et al. Splenic infarction in human babesiosis: two cases and discussion. Clin Infect Dis. 2008; 46(1): e8-11. PMid:18171204 http://dx.doi.org/10.1086/524081

[4] Wozniak EJ, Lowenstine LJ, Hemmer R, et al. Comparative pathogenesis of human WA1 and Babesia microti isolates in a Syrian hamster model. Lab Anim Sci. 1996; 46(5): 507-15. PMid:8905583

[5] Tobler WD, Cotton D, Lepore T, et al. Case Report: Successful nonoperative management of spontaneous splenic rupture in a patient with babesiosis. World J Emerg Surg. 2011; 6: 4. PMid:21251311 http://dx.doi.org/10.1186/1749-7922-6-4
[6] Kuwayama DP, Briones RJ. Spontaneous splenic rupture caused by Babesia microti infection. Clin Infect Dis. 2008; 46(9): e92-5. PMid:18419430 http://dx.doi.org/10.1086/587175

[7] Wormser GP, Dattwyler RJ, Shapiro ED, et al. The clinical assessment, treatment, and prevention of lyme disease, human granulocytic anaplasmosis, and babesiosis: clinical practice guidelines by the Infectious Diseases Society of America. Clin Infect Dis. 2006; 43(9): 1089-134. PMid:17029130 http://dx.doi.org/10.1086 /508667

[8] Krause PJ, Lepore T, Sikand VK, et al. Atovaquone and azithromycin for the treatment of babesiosis. N Engl J Med. 2000; 343(20): 1454-8. PMid:11078770 http://dx.doi.org/10.1056/NEJM2 00011163432004

[9] Weiss LM, Wittner M, Wasserman S, et al. Efficacy of azithromycin for treating Babesia microti infection in the hamster model. J Infect Dis. 1993; 168(5): 1289-92. PMid:8228366 http://dx.doi.org /10.1093/infdis/168.5.1289 